PHYSICAL REVIEW D 85, 075014 (2012)

\title{
Improved squark and gluino mass limits from searches for supersymmetry at hadron colliders
}

\author{
Wim Beenakker \\ Theoretical High Energy Physics, IMAPP Mailbox 79, P.O. Box 9010, NL-6500 GL Nijmegen, The Netherlands \\ Silja Brensing \\ DESY, Theory Group, Notkestrasse 85, D-22603 Hamburg, Germany \\ Monica D'Onofrio \\ University of Liverpool, Oliver Lodge Laboratory, P.O. Box 147, Oxford Street, Liverpool L69 3BX, United Kingdom \\ Michael Krämer and Anna Kulesza \\ Institute for Theoretical Particle Physics and Cosmology, RWTH Aachen University, D-52056 Aachen, Germany \\ Eric Laenen \\ ITFA, University of Amsterdam, Science Park 904, 1090 GL Amsterdam, The Netherlands, \\ and ITF, Utrecht University, Leuvenlaan 4, 3584 CE Utrecht Nikhef, Science Park 105, 1098 XG Amsterdam, The Netherlands \\ Mario Martinez \\ ICREA and Institut de Física dAltes Energies, IFAE, Edifici Cn, Universitat Autònoma de Barcelona, \\ ES - 08193 Bellaterra (Barcelona), Spain \\ Irene Niessen \\ Theoretical High Energy Physics, IMAPP Mailbox 79, P.O. Box 9010, NL-6500 GL Nijmegen, The Netherlands
}

(Received 30 June 2011; published 18 April 2012)

\begin{abstract}
Squarks and gluinos have been searched for at hadron colliders in events with multiple jets and missing transverse energy. No excess has been observed to date, and from a comparison of experimental cross section limits and theoretical cross section predictions one can deduce lower bounds on the squark and gluino masses. We present an improved analysis of squark and gluino mass bounds which is based on state-of-the-art cross section calculations including the summation of large threshold corrections. For illustration, we consider experimental data obtained by the CDF collaboration at the Fermilab Tevatron and discuss the impact of the improved cross section predictions on the squark and gluino mass limits.
\end{abstract}

DOI: 10.1103/PhysRevD.85.075014

PACS numbers: 14.80.Ly, 12.38.Cy

\section{INTRODUCTION}

The search for supersymmetry (SUSY) [1,2] is one of the central tasks at the currently operating hadron colliders Tevatron and LHC. In most SUSY models the squarks $(\tilde{q})$ and gluinos $(\tilde{g})$, the colored supersymmetric particles (sparticles), are produced copiously in hadronic collisions. We consider the minimal supersymmetric extension of the standard model (MSSM) [3,4] where, as a consequence of $R$-parity conservation, squarks and gluinos are produced in pairs and where the lightest supersymmetric particle (LSP) is stable. For large regions of parameter space the LSP is also neutral and weakly interacting, and provides a viable dark matter candidate. The expected MSSM signature at colliders is thus characterized by multiple jets from cascade decays of squarks and gluinos and by large missing transverse energy $E_{T \text {,miss }}$ from the two LSPs at the end of each decay chain.

Searches for squarks and gluinos at the protonantiproton collider Tevatron with a center-of-mass energy of $\sqrt{S}=1.96 \mathrm{TeV}$ have placed lower limits on squark and gluino masses in the range of 300-400 GeV [5,6] in a minimal supergravity (mSUGRA) scenario [7-10]. The proton-proton collider LHC currently operating at $\sqrt{S}=$ $7 \mathrm{TeV}$ has already significantly extended the mSUGRA mass limits to values near $800 \mathrm{GeV}$ [11,12]. The mass bounds are deduced from the comparison of experimental cross section exclusion limits and theoretical cross section predictions. So far, the interpretation of the experimental search results have relied on calculations of the squark and gluino cross sections at next-to-leading-order (NLO) in SUSY-QCD [13-16]. Recently, the accuracy of these cross section predictions has been improved further by the resummation of soft-gluon emission to all orders in perturbation theory [17-21]. The inclusion of softgluon resummation leads to a significant reduction of the unphysical renormalization and factorization-scale dependence and, in general, increases the NLO cross sections.

In this work we employ state-of-the-art SUSY-QCD cross section predictions including next-to-leadinglogarithmic (NLL) soft-gluon resummation [17-21] to 
derive improved mass bounds on squarks and gluinos from the analysis of recent CDF data [6].

The paper is structured as follows. In section II we briefly summarize the status of cross section calculations for squark and gluino hadroproduction and discuss the improvement obtained from soft-gluon resummation. The experimental CDF analysis is reviewed in Sec. III. The interpretation of the cross section limits in terms of squark and gluino mass bounds is discussed in Sec. IV, where we also address the treatment of theoretical uncertainties. We conclude in Sec. V.

\section{SQUARK AND GLUINO CROSS SECTIONS}

In the MSSM with $R$-parity conservation, squarks and gluinos are produced in pairs,

$$
p p / p \bar{p} \rightarrow \tilde{q} \overline{\tilde{q}}, \tilde{q} \tilde{q}, \tilde{q} \tilde{g}, \tilde{g} \tilde{g}+X .
$$

In Eq. (1) and throughout the rest of this paper we suppress the chiralities of the squarks $\tilde{q}=\left(\tilde{q}_{L}, \tilde{q}_{R}\right)$ and do not explicitly state the charge-conjugated processes. We include squarks $\tilde{q}$ of any flavor except for top squarks. The production of top squarks [16] needs to be considered separately because of potentially large mixing effects and mass splitting in the stop sector [22].

The cross sections for the squark and gluino-pairproduction processes (1) have been known at next-toleading-order in SUSY-QCD [13-16] for some time. Electroweak corrections to the $\mathcal{O}\left(\alpha_{\mathrm{s}}^{2}\right)$ tree-level production [23-27] and the electroweak Born production channels of $\mathcal{O}\left(\alpha \alpha_{\mathrm{s}}\right)$ and $\mathcal{O}\left(\alpha^{2}\right)$ [28,29] are significant for the pair production of $\mathrm{SU}(2)$-doublet squarks $\tilde{q}_{L}$ and at large invariant masses in general, but they are moderate for total cross sections summed over all squark species.

The NLO SUSY-QCD corrections to squark and gluino hadroproduction reduce the renormalization- and factorization-scale dependence of the predictions. In general the NLO contributions also significantly increase the cross section with respect to the leading-order (LO) approximation [30-32] if the renormalization and factorization scales are chosen close to the average mass of the pair-produced sparticles. A significant part of the NLO corrections can be attributed to the threshold region, where the partonic center-of-mass energy is close to the kinematic production threshold. In this region the NLO corrections are dominated by the contributions due to soft-gluon emission off the colored particles in the initial and final state and by the Coulomb corrections due to the exchange of gluons between the massive sparticles in the final state. The soft-gluon corrections can be taken into account to all orders in perturbation theory by means of threshold resummation techniques.

Recently, such threshold resummation has been performed for all squark and gluino production processes in Eq. (1) at NLL accuracy [17-20]. For squark-antisquark production, NLL resummation has also been addressed in the framework of effective field theories [33,34], and the dominant next-to-next-to-leading-order (NNLO) correction coming from the resummed cross section at next-tonext-to-leading-logarithmic (NNLL) level has been derived in Ref. [35,36]. Moreover, a formalism allowing for the resummation of soft and Coulomb gluons in the production of colored sparticles has been presented in Refs. [33,34], and bound state effects have been studied for gluino-pair production in Ref. [37].

In this work we will employ the state-of-the-art SUSYQCD predictions at NLO + NLL for squark and gluino production to derive mass bounds from the comparison with experimental exclusion limits. In the remainder of this section we briefly review the calculation of the NLO + NLL cross section presented in [17-21] and present a few illustrative numerical results.

The hadronic threshold for inclusive production of two final-state particles with masses $m_{k}$ and $m_{l}$ corresponds to a hadronic center-of-mass energy squared that is equal to $S=\left(m_{k}+m_{l}\right)^{2}$. Thus we define the threshold variable $\rho$, measuring the distance from threshold in terms of energy fraction, as

$$
\rho=\frac{\left(m_{k}+m_{l}\right)^{2}}{S}
$$

Our results are based on the following expression for the NLL-resummed cross section matched to the exact NLO calculation of Refs. [13-15],

$$
\begin{aligned}
\sigma_{h_{1} h_{2} \rightarrow k l}^{(\mathrm{NLO}+\mathrm{NLL})}\left(\rho,\left\{m^{2}\right\}, \mu^{2}\right) \\
=\sigma_{h_{1} h_{2} \rightarrow k l}^{\mathrm{NNO})}\left(\rho,\left\{m^{2}\right\}, \mu^{2}\right) \\
\quad+\frac{1}{2 \pi i} \sum_{i, j=q, \bar{q}, g} \int_{\mathrm{CT}} d N \rho^{-N} \tilde{f}_{i / h_{1}}\left(N+1, \mu^{2}\right) \tilde{f}_{j / h_{2}}\left(N+1, \mu^{2}\right) \\
\quad \times\left[\tilde{\sigma}_{i j \rightarrow k l}^{\text {(res }}\left(N,\left\{m^{2}\right\}, \mu^{2}\right)-\left.\tilde{\sigma}_{i j \rightarrow k l}^{\text {(res) }}\left(N,\left\{m^{2}\right\}, \mu^{2}\right)\right|_{(\mathrm{NLO})}\right],
\end{aligned}
$$

where the last term in the square brackets denotes the NLLresummed expression expanded to NLO. The initial state hadrons are denoted generically as $h_{1}$ and $h_{2}$, while $\mu$ is the common renormalization and factorization scale. The resummation is performed after taking a Mellin transform (indicated by a tilde) of the cross section,

$$
\tilde{\sigma}_{h_{1} h_{2} \rightarrow k l}\left(N,\left\{m^{2}\right\}\right) \equiv \int_{0}^{1} d \rho \rho^{N-1} \sigma_{h_{1} h_{2} \rightarrow k l}\left(\rho,\left\{m^{2}\right\}\right) .
$$

To evaluate the contour CT of the inverse Mellin transform in Eq. (3) we adopt the "minimal prescription" of Ref. [38]. The NLL-resummed cross section in Eq. (3) reads 


$$
\begin{aligned}
& \tilde{\sigma}_{i j \rightarrow k l}^{(\mathrm{res})}\left(N,\left\{m^{2}\right\}, \mu^{2}\right) \\
& =\sum_{I} \tilde{\sigma}_{i j \rightarrow k l, I}^{(0)}\left(N,\left\{m^{2}\right\}, \mu^{2}\right) C_{i j \rightarrow k l, I}\left(N,\left\{m^{2}\right\}, \mu^{2}\right) \\
& \quad \times \Delta_{i}\left(N+1, Q^{2}, \mu^{2}\right) \Delta_{j}\left(N+1, Q^{2}, \mu^{2}\right) \\
& \quad \times \Delta_{i j \rightarrow k l, I}^{(s)}\left(N+1, Q^{2}, \mu^{2}\right),
\end{aligned}
$$

where we have introduced the hard scale $Q^{2}=$ $\left(m_{k}+m_{l}\right)^{2}$. The color-decomposed leading-order cross sections in Mellin-moment space are denoted by $\tilde{\sigma}_{i j \rightarrow k l, I}^{(0)}$
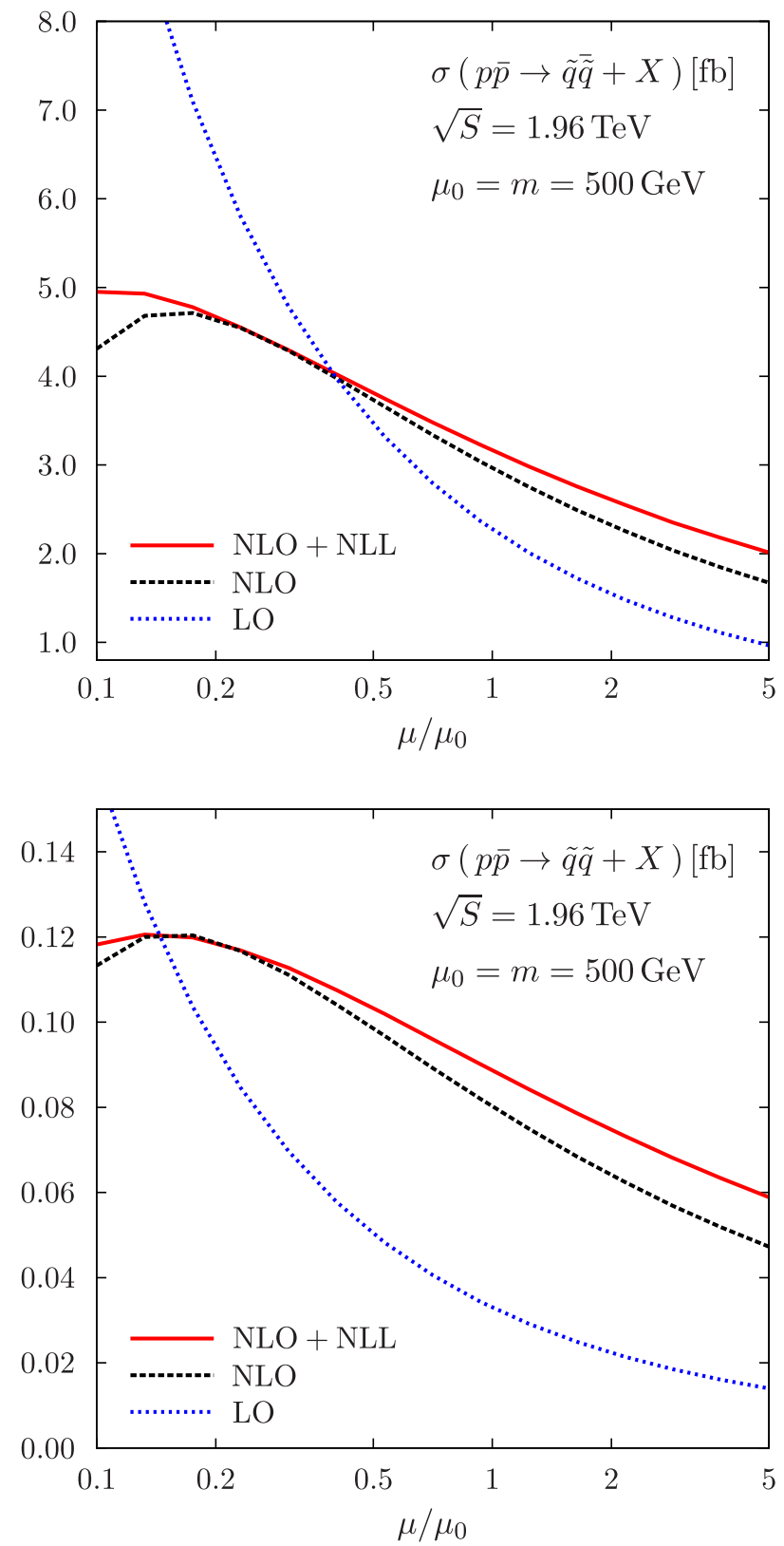

with $I$ labeling the different possible color structures. The expressions for these, both in moment and in momentum space, can be found in $[18,19]$. The perturbative functions $C_{i j \rightarrow k l, I}$ contain information about hard contributions beyond leading order. This information is only relevant beyond NLL accuracy and therefore we keep $C_{i j \rightarrow k l, I}=1$ in our calculations. The functions $\Delta_{i}$ and $\Delta_{j}$ sum the effects of the (soft-)collinear radiation from the incoming partons. They are process independent and do not depend on the color structures. They contain the leading logarithmic dependence, as well as part of the subleading logarithmic
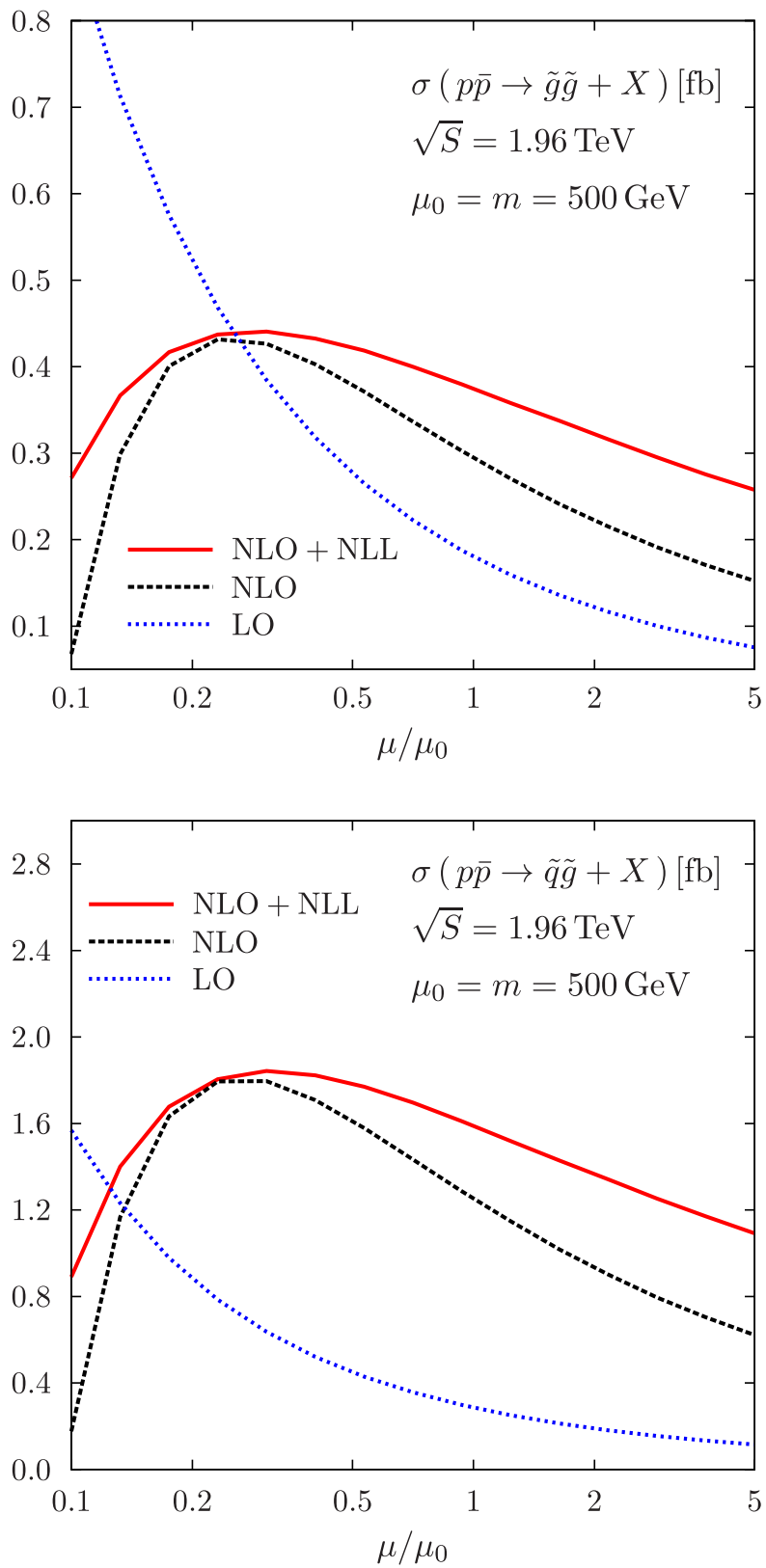

FIG. 1 (color online). The scale dependence of the LO, NLO, and NLO + NLL cross sections for the four different squark and gluino production processes at the Tevatron. The squark and gluino masses have been set to $m_{\tilde{q}}=m_{\tilde{g}}=m=500 \mathrm{GeV}$. The CTEQ6.6 [41] PDF has been adopted. 
behavior, and are listed e.g. in Ref. [18]. The resummation of the soft-gluon contributions, which does depend on the color structures in which the final-state SUSY particle pairs can be produced, contributes at the NLL level and is summarized by the factor

$$
\Delta_{i j \rightarrow k l, I}^{(\mathrm{s})}\left(Q /(N \mu), \mu^{2}\right)=\exp \left[\int_{\mu}^{Q / N} \frac{d q}{q} \frac{\alpha_{\mathrm{s}}(q)}{\pi} D_{I}\right] .
$$

The one-loop coefficients $D_{I}$ are derived and listed in $[18,19]$.

We now present a few selected numerical results to illustrate the impact of the NLL resummation on squark and gluino production at the Tevatron. We compare the LO, NLO, and NLO + NLL matched results and discuss the theoretical uncertainty due to the choice of renormalization and factorization scales, and the parametrization of the parton distribution functions (PDFs). The NLO cross sections have been calculated in Refs. [13-16] and are available in the form of the public computer code PROSPINO [39]. The $\overline{\mathrm{MS}}$-scheme with five active flavors is used to define the QCD coupling $\alpha_{\mathrm{s}}$ and the parton distribution functions at NLO. The masses of the squarks and gluinos are renormalized in the on-shell scheme, and the SUSY particles are decoupled from the running of $\alpha_{\mathrm{s}}$ and the PDFs.

We first discuss the scale dependence of the SUSY-QCD cross section prediction for the four different production processes $p \bar{p} \rightarrow \tilde{q} \overline{\tilde{q}}, \tilde{q} \tilde{q}, \tilde{q} \tilde{g}, \tilde{g} \tilde{g}+X$ at the Tevatron. For convenience we define the average mass of the final-state sparticle pair $m=\left(m_{k}+m_{l}\right) / 2$, which reduces to the squark and gluino mass for $\tilde{q} \overline{\tilde{q}}, \tilde{q} \tilde{q}$ and $\tilde{g} \tilde{g}$ final states, respectively. The renormalization and factorization scales are taken to be equal. Figure 1 compares the scale dependence in LO, NLO, and NLO + NLL for $m_{\tilde{q}}=m_{\tilde{g}}=m=$ $500 \mathrm{GeV}$. The scale $\mu$ is varied around the standard scale choice $\mu_{0}=m$ from $\mu=\mu_{0} / 10$ up to $\mu=5 \mu_{0}$. Note that the LO predictions are obtained with LO PDFs and the corresponding LO values for $\alpha_{\mathrm{s}}$. We observe the anticipated strong reduction of the scale dependence when going

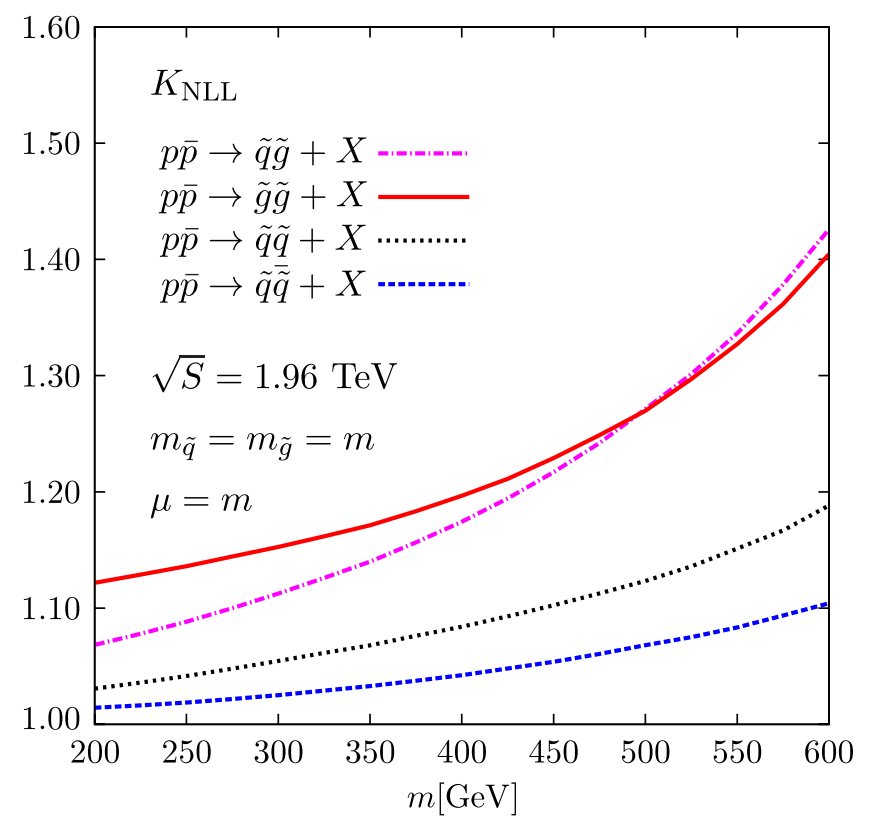

FIG. 2 (color online). The NLL $K$-factor $K_{\mathrm{NLL}}=$ $\sigma_{\mathrm{NLO}+\mathrm{NLL}} / \sigma_{\mathrm{NLO}}$ for squark and gluino-pair-production processes at the Tevatron as a function of the average sparticle mass $m$. The CTEQ6.6 [41] PDF has been adopted.

from LO to NLO, and a further significant improvement when the resummation of threshold logarithms is included, in particular, for $\tilde{g} \tilde{g}$ and $\tilde{q} \tilde{g}$ production.

At the standard choice of scale $\mu=\mu_{0}=m$ the cross section predictions are in general enhanced by soft-gluon resummation. The NLL $K$-factor $K_{\mathrm{NLL}} \equiv \sigma_{\mathrm{NLO}+\mathrm{NLL}} /$ $\sigma_{\mathrm{NLO}}$ at the Tevatron is displayed in Fig. 2 for squark and gluino masses in the range between $200 \mathrm{GeV}$ and $600 \mathrm{GeV}$. We show results for equal squark and gluino masses, $K$-factors for different mass ratios $m_{\tilde{g}} / m_{\tilde{q}}$ can be found in Ref. [19]. The soft-gluon corrections are moderate for $\tilde{q} \overline{\tilde{q}}$ production, but very significant for $\tilde{g} \tilde{g}, \tilde{q} \tilde{q}$ and $\tilde{q} \tilde{g}$ final states, respectively. Because of the increasing importance of the threshold region, the corrections become in

TABLE I. The LO, NLO, and NLO + NLL cross sections for inclusive sparticle pair production $p \bar{p} \rightarrow \tilde{q} \overline{\tilde{q}}+\tilde{q} \tilde{q}+\tilde{q} \tilde{g}+\tilde{g} \tilde{g}+X$ at the Tevatron $(\sqrt{S}=1.96 \mathrm{TeV})$, including errors due to scale variation $\left(\Delta \sigma_{\mu}\right)$ in the range $m / 2 \leq \mu \leq 2 m$. Results are shown for two PDF parametrizations (CTEQ6.1 [40], CTEQ6.6 [41]) with the corresponding 68\% C.L. PDF error estimates.

\begin{tabular}{|c|c|c|c|c|}
\hline \multirow[b]{2}{*}{$m_{\tilde{q}} / m_{\tilde{g}}(\mathrm{GeV})$} & \multicolumn{2}{|c|}{$\begin{array}{c}p \bar{p} \rightarrow \tilde{q} \overline{\tilde{q}}+\tilde{q} \tilde{q}+\tilde{q} \tilde{g}+\tilde{g} \tilde{g}+X \text { at } \sqrt{S}=1.96 \mathrm{TeV} \\
\text { CTEQ6.1 }\end{array}$} & \multicolumn{2}{|c|}{ CTEQ6.6 } \\
\hline & $400 / 400$ & $460 / 300$ & $400 / 400$ & $460 / 300$ \\
\hline$\overline{\left(\sigma \pm \Delta \sigma_{\mu}\right)_{\mathrm{LO}}[\mathrm{pb}]}$ & $\left(5.39_{-1.66}^{+2.66}\right) \times 10^{-2}$ & $\left(1.51_{-0.44}^{+0.71}\right) \times 10^{-1}$ & $\left(5.39_{-1.66}^{+2.66}\right) \times 10^{-2}$ & $\left(1.51_{-0.44}^{+0.71}\right) \times 10^{-1}$ \\
\hline$\left(\sigma \pm \Delta \sigma_{\mu}\right)_{\mathrm{NLO}}[\mathrm{pb}]$ & $\left(8.83_{-1.82}^{+1.96}\right) \times 10^{-2}$ & $\left(3.41_{-0.76}^{+0.90}\right) \times 10^{-1}$ & $\left(8.49_{-1.73}^{+1.85}\right) \times 10^{-2}$ & $\left(3.16_{-0.69}^{+0.81}\right) \times 10^{-1}$ \\
\hline$\left(\sigma \pm \Delta \sigma_{\mu}\right)_{\mathrm{NLO}+\mathrm{NLL}}[\mathrm{pb}]$ & $\left(9.71_{-1.45}^{+1.44}\right) \times 10^{-2}$ & $\left(3.77_{-0.60}^{+0.68}\right) \times 10^{-1}$ & $\left(9.30_{-1.38}^{+1.37}\right) \times 10^{-2}$ & $\left(3.47_{-0.54}^{+0.62}\right) \times 10^{-1}$ \\
\hline$\Delta \operatorname{pdf}_{\mathrm{NLO}}[\%]$ & $\begin{array}{l}+26 \\
-14\end{array}$ & $\begin{array}{l}+32 \\
-18\end{array}$ & $\begin{array}{r}+16 \\
-10\end{array}$ & $\begin{array}{l}+19 \\
-13\end{array}$ \\
\hline$K_{\mathrm{NLO}}$ & 1.64 & 2.26 & 1.58 & 2.09 \\
\hline$K_{\mathrm{NLL}}$ & 1.10 & 1.11 & 1.10 & 1.10 \\
\hline
\end{tabular}


general larger for increasing sparticle masses. The large effect of soft-gluon resummation for $\tilde{q} \tilde{g}$ and $\tilde{g} \tilde{g}$ production can be mostly attributed to the importance of gluon initial states for these processes. Furthermore, the presence of gluinos in the final-state results in an enhancement of the NLL contributions since the Casimir invariants which enter the resummation formulae are larger than for processes involving only squarks. The substantial value of $K_{\mathrm{NLL}}$ for $\tilde{q} \tilde{q}$ production at the Tevatron is a consequence of the behavior of the corresponding NLO corrections, which strongly decrease with increasing squark mass [15].

Representative values for the LO, NLO, and NLO + NLL cross sections are collected in Table I. The CDF analysis [6] was based on cross section predictions obtained with the CTEQ6.1 [40] PDF set. In Table I we thus compare predictions for the old CTEQ6.1 PDF and for the more recent CTEQ6.6 [41] PDF, which we use to derive the improved cross section limits presented in this paper.

As discussed before, we observe an increase of the cross section prediction near the central scale when going from LO to NLO and a further enhancement when NLL threshold resummation is included. The scale dependence in the range $m / 2 \leq \mu \leq 2 m$ is reduced from about $\pm 25 \%$ at NLO to about $\pm 15 \%$ at NLO + NLL. The estimated PDF uncertainty is approximately $15 \%$. The cross section is reduced when going from the old CTEQ6.1 [40] to the more modern CTEQ6.6 [41] PDF set.

\section{EXPERIMENTAL SEARCHES FOR SQUARKS AND GLUINOS}

Experimental searches for inclusive squark and gluino production are based on the study of events with multiple jets of hadrons and large $E_{T \text {,miss }}$ in the final state. In the CDF study [6] at the Tevatron, the SUSY samples were generated using the ISASUGRA implementation in PYTHIA [42] and normalized according to NLO cross sections as determined using PROSPINO 2.0 with CTEQ6.1M PDFs and the renormalization and factorization scales set equal to the average mass of the sparticles produced in the hard interaction. The uncertainties in the NLO predictions due to PDFs varied between $10 \%$ and $25 \%$ across the region of the squark/gluino mass plane considered, and variations by a factor two of the renormalization and factorization scales changed the theoretical predictions by $20 \%$ to $25 \%$. Final states with different inclusive jet multiplicity were considered to optimize the sensitivity across the quark/gluino mass plane. In the scenario with squark masses significantly larger than the gluino mass at least four jets in the final state are expected, while for a gluino mass much larger than the squark masses dijet configurations dominate. In the CDF analysis, three parallel analyses in event topologies with large $E_{T \text {,miss }}$ and at least four, three, and two jets in the final state were carried out. In each case the event selection criteria were optimized such as to maximize the sensitivity to the SUSY signal. As an example,
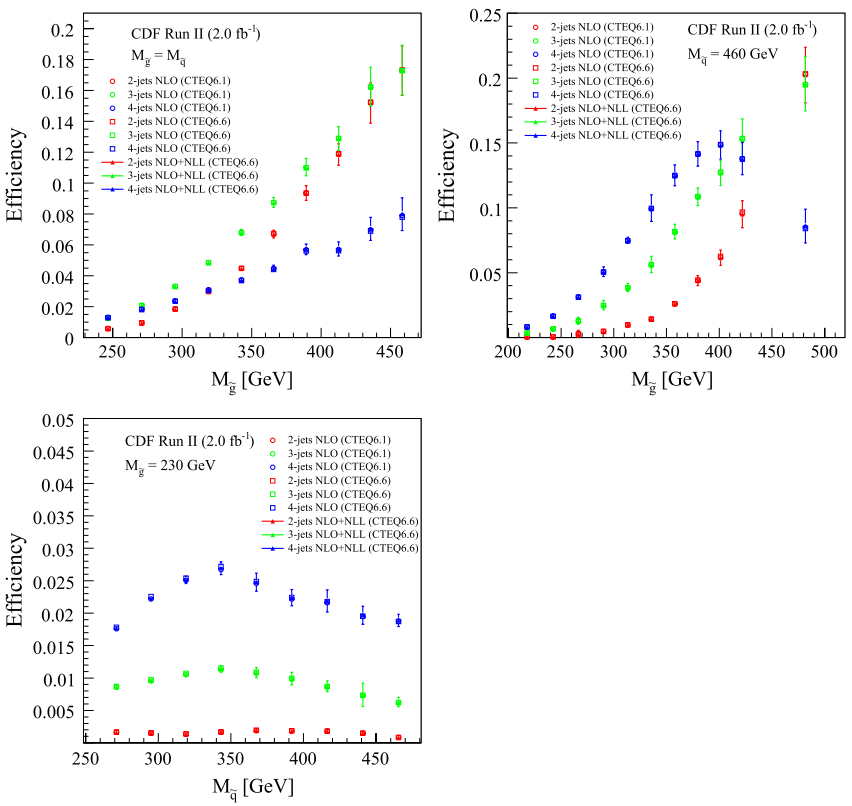

FIG. 3 (color online). SUSY signal selection efficiencies for different inclusive jet multiplicities, as determined using PYTHIA Monte Carlo samples normalized according to NLO cross sections with CTEQ6.1M [40] and CTEQ6.6 [41] PDFs, or using NLO + NLL cross sections with CTEQ6.6 PDFs. Different squark and gluino mass configurations are considered and the efficiencies are presented as a function of squark and gluino masses.

for degenerate squarks and gluinos with masses around $400 \mathrm{GeV}, S / \sqrt{B}=6^{1}$ was obtained, with a signal efficiency, defined as the fraction of signal events passing the selection criteria, of about $12 \%$. Additional details on the CDF data analysis can be found in $[43,44]$. In this paper, CDF results are revisited with improved NLO + NLL theoretical predictions, leading to reduced scale uncertainties, and updated CTEQ6.6 PDF sets.

A variation in the theoretical predictions can potentially translate into a change in the signal selection efficiency if the mixture among subprocesses contributing to the same final state is modified significantly. The effect of updated PDFs and improved NLO + NLL predictions is considered separately, and the variation of the signal selection efficiency is studied as a function of the squark and gluino masses in three different configurations already considered by CDF [44]: degenerate squarks and gluinos, fixed squark mass $m_{\tilde{q}}=460 \mathrm{GeV}$, and fixed gluino mass $m_{\tilde{g}}=$ $230 \mathrm{GeV}$. Figure 3 shows the results separately for each jet multiplicity. In all cases, the changes in the signal selection efficiencies are small, indicating that the updated theoretical predictions do not introduce a significant variation.

\footnotetext{
${ }^{1}$ Here $S$ and $B$ denote the number of signal and SM background events, respectively.
} 

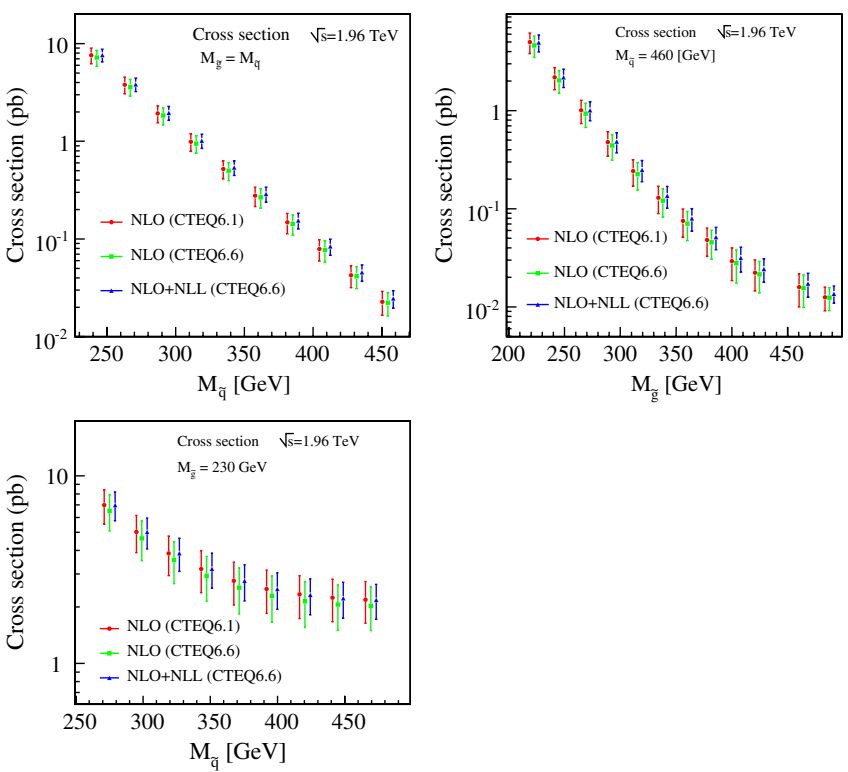

FIG. 4 (color online). NLO cross section prediction using CTEQ6.1M [40] and CTEQ6.6 [41] PDFs, and NLO + NLL cross sections with CTEQ6.6 PDFs. Different squark and gluino mass configurations are considered and the predictions are presented as a function of squark and gluino masses. The error bars indicate the PDF and renormalization and factorization scales added in quadrature. Note that for display purposes the NLO and NLO + NLL CTEQ6.6 cross sections are shown at mass coordinates shifted by $4 \mathrm{GeV}$ and $8 \mathrm{GeV}$, respectively, with respect to the NLO CTEQ6.1 cross section.

The new predictions for the SUSY cross sections in the different squark/gluino mass configurations considered above are compared to those used by CDF in Fig. 4. Again, the impact of the PDF set employed and the inclusion of the new NLO + NLL terms are studied separately. In general, the use of the CTEQ6.6 PDFs slightly reduces the theoretical cross sections, whereas the NLO + NLL calculations predict an enhanced SUSY signal cross section with a reduced scale dependence. As a result, the new predictions differ from the previous ones by less than $1 \%$ across the different squark and gluino masses considered, but present smaller uncertainties. Only in the scenario of similar squark and gluino masses, the new SUSY cross sections are significantly larger, and the difference varies from $0.3 \%$ to $10 \%$ with increasing mass between $250 \mathrm{GeV}$ and $460 \mathrm{GeV}$.

\section{SQUARK AND GLUINO EXPERIMENTAL BOUNDS}

The effect of the new improved theoretical cross sections on the calculation of the $95 \%$ confidence level (C.L.) upper limits for SUSY production cross sections and squark and gluino masses is investigated. In a counting experiment, the Poisson probability of observing $n$ events with an expected background $b$ and a signal efficiency $\epsilon$ is

$$
\frac{e^{-(L s \epsilon+b)}(L s \epsilon+b)^{n}}{n !},
$$

where $L$ is the integrated luminosity and $s$ is the signal cross section. Following the original CDF study, a Bayesian approach [45] is adopted to compute the 95\% C.L. upper limits on $s$ given the observed number of events in the data. The calculation includes statistical and systematic uncertainties on $\epsilon$ and $b$ and their correlations, and a $6 \%$ uncertainty on $L$. In order to compute the mass bounds, the theoretical uncertainties on $s$ are also included in the limit calculation via an extra uncertaity on $\epsilon$. For each mSUGRA point considered, observed and expected limits are computed separately for each of the three analyses, corresponding to different jet multiplicities in the final state, and the one with the best expected limit is adopted as the nominal result.

Figure 5 shows, in the case of degenerate squarks and gluinos, the new expected and observed 95\% C.L. upper limits on the production cross section compared to previous results. As anticipated from the observation of nearly invariant signal efficiencies after the inclusion of the NLL terms in the calculation, the obtained cross section upper limits are close to those published by CDF. The results on the new squark and gluino mass bounds are presented in Fig. 6 for the whole squark/gluino mass plane. The impact from the new theoretical predictions on the squark and

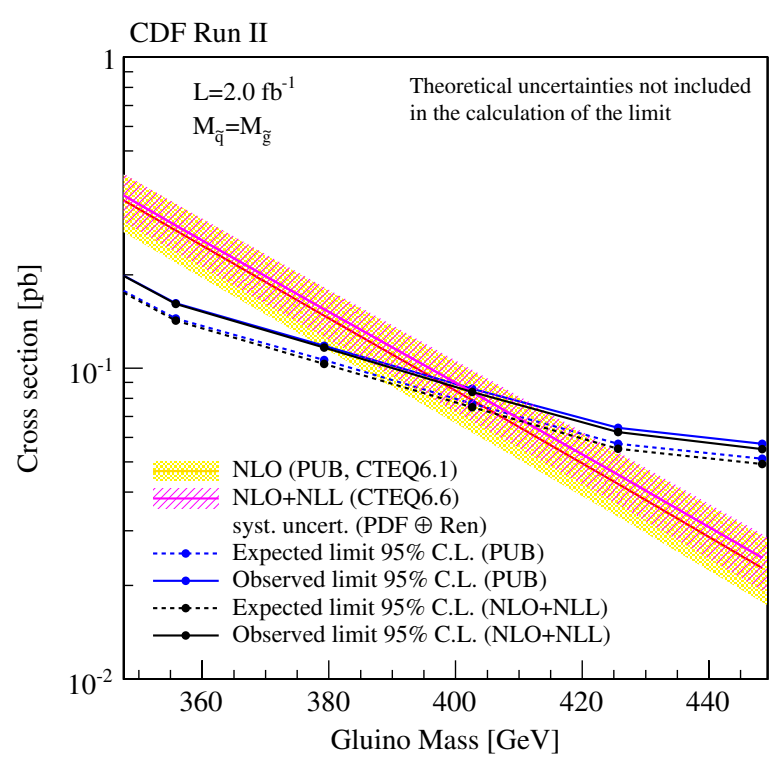

FIG. 5 (color online). Expected and observed 95\% C.L. upper limit on the SUSY production cross section in the case of degenerate squarks and gluinos. The new limits (using NLO + NLL and CTEQ6.6 PDFs theoretical predictions) are compared to previous CDF published results [6]. 


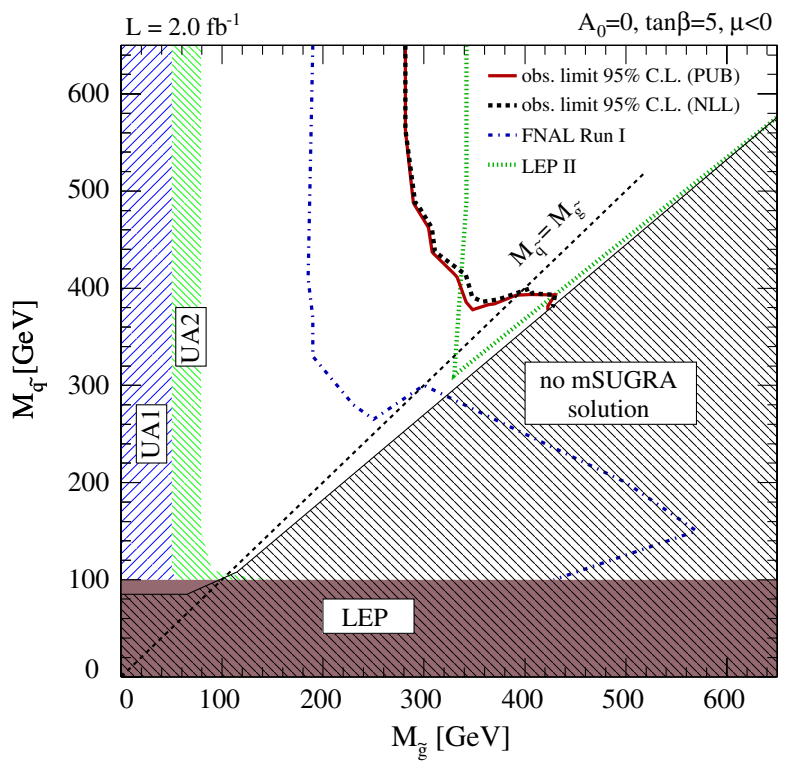

FIG. 6 (color online). Observed 95\% C.L. exclusion region in the squark/gluino mass plane. The new results (dashed line) are compared to CDF published results [6] (solid line).

gluino mass limits is modest and only relevant for the case of similar squark and gluino masses, extending the CDF excluded region by 5 to $10 \mathrm{GeV}$.

\section{CONCLUSIONS}

Precise theoretical predictions for sparticle cross sections are essential for the interpretation of current and future searches for supersymmetry at hadron colliders. Recently, the NLO-QCD cross section calculation has been further improved by the inclusion of threshold resummation at NLL accuracy. The NLL corrections reduce the renormalization- and factorization-scale dependence of the predictions. In general the NLL contributions also increase the cross section if the renormalization and factorization scales are chosen close to the average mass of the pair-produced sparticles.

The impact of the new improved theoretical NLO + NLL calculations on the experimental bounds for squark and gluino production has been shown using CDF results as benchmark. Improved 95\% C.L. upper limits on the SUSY production cross section and the squark and gluino masses are presented in the case of nearly degenerate squarks and gluinos. The difference with respect to the CDF published bounds are found to be small once the new NLO + NLL cross section predictions are combined with updated CTEQ6.6 PDF sets.

\section{ACKNOWLEDGMENTS}

The work has been supported by the Helmholtz Alliance "Physics at the Terascale," the DFG Graduiertenkolleg "Elementary Particle Physics at the TeV Scale," the Foundation for Fundamental Research of Matter (FOM) program "Theoretical Particle Physics in the Era of the LHC," the National Organization for Scientific Research (NWO), the DFG SFB/TR9 "Computational Particle Physics," and the European Community's Marie-Curie Research Training Network under Contract No. MRTNCT-2006-035505 "Tools and Precision Calculations for Physics Discoveries at Colliders." M. K. would like to thank the Institute for High Energy Physics (IFAE) at the Universitat Autónoma de Barcelona for hospitality and the Deutsche Forschungsgemeinschaft (DFG) for financial support.
[1] Yu. A. Golfand and E. P. Likhtman, Pis'ma Zh. Eksp. Teor. Fiz. 13, 452 (1971) [JETP Lett. 13, 323 (1971)].

[2] J. Wess and B. Zumino, Nucl. Phys. B70, 39 (1974).

[3] H. P. Nilles, Phys. Rep. 110, 1 (1984).

[4] H. E. Haber and G. L. Kane, Phys. Rep. 117, 75 (1985).

[5] V. M. Abazov et al. (D0 Collaboration), Phys. Lett. B 660, 449 (2008).

[6] T. Aaltonen et al. (CDF Collaboration), Phys. Rev. Lett. 102, 121801 (2009).

[7] H. P. Nilles, Phys. Lett. B 115, 193 (1982).

[8] H. P. Nilles, Nucl. Phys. B217, 366 (1983).

[9] A. H. Chamseddine, R. L. Arnowitt, and P. Nath, Phys. Rev. Lett. 49, 970 (1982).

[10] R. Barbieri, S. Ferrara, and C. A. Savoy, Phys. Lett. B 119, 343 (1982)

[11] V. Khachatryan et al. (CMS Collaboration), Phys. Lett. B 698, 196 (2011).
[12] G. Aad et al. (Atlas Collaboration), Phys. Rev. Lett. 106, 131802 (2011).

[13] W. Beenakker, R. Höpker, M. Spira, and P. M. Zerwas, Phys. Rev. Lett. 74, 2905 (1995).

[14] W. Beenakker, R. Höpker, M. Spira, and P. M. Zerwas, Z. Phys. C 69, 163 (1995).

[15] W. Beenakker, R. Höpker, M. Spira, and P. M. Zerwas, Nucl. Phys. B492, 51 (1997).

[16] W. Beenakker, M. Krämer, T. Plehn, M. Spira, and P. M. Zerwas, Nucl. Phys. B515, 3 (1998).

[17] A. Kulesza and L. Motyka, Phys. Rev. Lett. 102, 111802 (2009).

[18] A. Kulesza and L. Motyka, Phys. Rev. D 80, 095004 (2009).

[19] W. Beenakker, S. Brensing, M. Krämer, A. Kulesza, E. Laenen, and I. Niessen, J. High Energy Phys. 12 (2009) 041. 
[20] W. Beenakker, S. Brensing, M. Krämer, A. Kulesza, E. Laenen, and I. Niessen, J. High Energy Phys. 08 (2010) 098.

[21] W. Beenakker, S. Brensing, M. Krämer, A. Kulesza, E. Laenen, L. Motyka, and I. Niessen, Int. J. Mod. Phys. A 26, 2637 (2011).

[22] J. R. Ellis and S. Rudaz, Phys. Lett. B 128, 248 (1983).

[23] W. Hollik, M. Kollar, and M. K. Trenkel, J. High Energy Phys. 02 (2008) 018.

[24] M. Beccaria, G. Macorini, L. Panizzi, F. M. Renard, and C. Verzegnassi, Int. J. Mod. Phys. A 23, 4779 (2008).

[25] W. Hollik and E. Mirabella, J. High Energy Phys. 12 (2008) 087.

[26] W. Hollik, E. Mirabella, and M. K. Trenkel, J. High Energy Phys. 02 (2009) 002.

[27] E. Mirabella, J. High Energy Phys. 12 (2009) 012.

[28] A. T. Alan, K. Cankocak, and D. A. Demir, Phys. Rev. D 75, 095002 (2007); 76, 119903(E) (2007).

[29] S. Bornhauser, M. Drees, H. K. Dreiner, and J. S. Kim, Phys. Rev. D 76, 095020 (2007).

[30] G. L. Kane and J. P. Leveille, Phys. Lett. B 112, 227 (1982).

[31] P. R. Harrison and C.H. Llewellyn Smith, Nucl. Phys. B213, 223 (1983); B223, 542 (1983).

[32] S. Dawson, E. Eichten, and C. Quigg, Phys. Rev. D 31, 1581 (1985).

[33] M. Beneke, P. Falgari, and C. Schwinn, Nucl. Phys. B828, 69 (2010).
[34] M. Beneke, P. Falgari, and C. Schwinn, Proc. Sci., EPSHEP2009 (2009) 319.

[35] U. Langenfeld and S. O. Moch, Phys. Lett. B 675, 210 (2009).

[36] U. Langenfeld, J. High Energy Phys. 07 (2011) 052.

[37] K. Hagiwara and H. Yokoya, J. High Energy Phys. 10 (2009) 049.

[38] S. Catani, M. L. Mangano, P. Nason, and L. Trentadue, Nucl. Phys. B478, 273 (1996).

[39] See http://www.thphys.uni-heidelberg.de/ plehn/prospino/ or http://people.web.psi.ch/spira/prospino/.

[40] D. Stump, J. Huston, J. Pumplin, W. K. Tung, H. L. Lai, S. Kuhlmann, and J.F. Owens, J. High Energy Phys. 10 (2003) 046 .

[41] P. M. Nadolsky, H.-L. Lai, Q.-H. Cao, J. Huston, J. Pumplin, D. Stump, W.-K. Tung, and C.-P. Yuan, Phys. Rev. D 78, 013004 (2008).

[42] T. Sjostrand, P. Eden, C. Friberg, L. Lonnblad, G. Miu, S. Mrenna, and E. Norrbin, Comput. Phys. Commun. 135, 238 (2001).

[43] G. De Lorenzo, Ph.D. thesis, Universitat Autonoma de Barcelona, 2010, Report No. FERMILAB-THESIS-201028.

[44] See also http://www.cdf.fnal.gov/physics/exotic/r2a/ 20080214.squark_gluino/squark_gluino.html.

[45] R. Cousins, Am. J. Phys. 63, 398 (1995).J. Heinrich C. Blocker, J. Conway, L. Demortier, L. Lyons, G. Punzi, and P. K. Sinervo, arXiv:physics/0409129v1. 